\title{
A Century of Progress in Evolutionary Genetics
}

\author{
Elements of Evolutionary Genetics, by Brian Charlesworth and Deborah Charlesworth. \\ Greenwood Village: Roberts and Company Publishers, 2010, pp. xxii + 734, H/b \$65.99
}

\author{
Diddahally R. Govindaraju
}

Published online: 1 May 2012

(C) Springer Science+Business Media, LLC 2012

In the very first three decades after the rediscovery of Mendelian Laws of heredity, a small group of biologists identifying themselves as geneticists, applied Mendelian principles to confirm or refute Darwin and Wallace's theory of natural selection. Like the salt doll, who in a proverbial Indian tale, dives into an ocean to determine its depth, only to dissolve away quickly, genetics was effectively absorbed into mainstream evolutionary thought by the 1930s via the modern synthesis. Although the basic tenets of the synthesis have remained relatively unchanged in the ensuing decades, many fundamental theoretical and experimental studies have confirmed as well as elaborated the ideas expounded in the modern synthesis. More importantly, introduction of molecular techniques in the 1960s has enriched and advanced both evolutionary biology and genetics, simply evolutionary genetics, in leaps and bounds. The authors of the present book, Brian and Deborah Charlesworth entered the evolutionary genetics scene between the late 1960s and the early 1970s at the cusp of what I call the second phase of the modern synthesis. Ever since, they have remained not only important players in the field, but also have contributed significantly to its growth through their theoretical and empirical studies on organisms with diverse life histories. The Charlesworths have now synthesized major advances in evolutionary genetics, spanning over a century, in the pages of their magnificent book-Elements of evolutionary genetics.

The book consists of ten chapters. The introduction provides a road map to the entire book and ends with an appendix, answers to problems from each of the chapters,

D. R. Govindaraju $(\bowtie)$

Beth Israel Medical Center and Harvard Medical School, Boston, MA, USA

e-mail: drgrajugis@gmail.com an extensive list of references, and author and subject index, resulting in a 734 page treatise. Each of these chapters begins with a summary of what to expect in the chapter, often followed by a quote from the early masters such as Darwin, Fisher, Haldane, Wright, etc., and ends with a set of problems. Some of these problems are worthy of pursuing as independent research projects. Mathematical details are placed in boxes, and approaches to understand them are presented in the appendix. A majority of these concepts are accessible with some effort; but a few of them, particularly diffusion equations, are daunting to most biologists, including this reviewer. This does not, however, interrupt the flow of thoughts or clarity of the presentation of the central concepts. The technical details are augmented by high-quality illustrations and graphs with clear accompanying explanations.

The landscape of the book is broad, the scholarship is breathtakingly brilliant and comprehensive, as its pendulum swings between Darwin-Wallace's theory and discoveries from the present-day genome sequencing studies, with attendant theoretical and experimental details. Results from classical and more contemporary genetic discoveries on organisms with diverse life histories and their basic similarities mingle effortlessly and weave together seamlessly. The very first figure on page 5 illustrates this point well; both classical and molecular aspects of inversions are depicted next to each other. Similarly, on page 289 , they state the similalrities between one of the most popularly used measure of molecular divergence, the Tajima's $D$ and the classical $t$ test. In a similar vein, distribution of quantitative and molecular genetic variation in plants and classic investigations on molecular variation in the Drosophila genome are also presented. Note that early molecular studies in the mid-1980s on Drosophila became the harbingers of the Human Genome Project, the HapMap project, as well as 
investigations into structural genome variation and genome sequences of numerous organisms. They also show that extensions of evolutionary genetics may also provide fundamental insights toward designing superior empirical studies to understand the genetic basis of human health. For instance, on page 295 , they suggest that "much human susceptibility to disease may be the effects of rare mutations with minor phenotypic effects," which run counter to the basic assumptions underlying the current genome wide association studies. Such important conceptual ideas are often ignored by the current crop of students and researchers, particularly in human genetics is experiencing unprecedented advances and also hitting unforeseen icebergs. Misunderstanding or omission of the very basic evolutionary genetic concepts could prove costly. Hence, in their discussion, the Charlesworths do not hesitate to mention the non-existent "sharp boundaries" among certain classes of polymorphisms including repeat number polymorphisms, microsatellites, and structural variants such as copy number variants (but presented in numerous journal articles). Similar even-handed treatment is found among the other chapters that deal with the origin and distribution of genetic variation. Occasionally, they even chastise biologists for misunderstanding important concepts or making blanket statements without paying attention to specific evolutionary factors, stating that, "Many biologists who have not been trained in evolutionary biology and even some who have, assume that a trait will evolve if it enhances the fitness of the population or the species as a whole, without asking whether it can spread through a population by the type of selection..." (p. 362).

In many ways, this is a unique book, and nothing quite like this exists now nor has existed before. Most other works that deal with similar topics do lip service to plant evolutionary genetics as well as the genetic basis of plant and animal breeding, or else they are completely omitted. This book, on the other hand, deals with not only all the major branches of evolutionary genetics (i.e., population, quantitative, molecular genetics, and evolution) but also presents almost all the classic and recent work done on these aspects in three diverse groups of organisms - microbes, animals, and plants. The authors' thorough treatment of the relationship among genetic load, inbreeding depression at single and multiple sites in the genome as well as fitness in both plants and animals, is rarely found among similar books. The famous debate between Fisher and Wright on the maintenance of dominance is clearly explained and its merit evaluated on the bases of recent experimental work. Similarly, some conjectures and other cryptic ideas of Fisher and Haldane are clarified. Evolution of breeding systems and self-incompatibility mechanisms that are so central to plant diversity are clearly presented. Their chapter on evolution in age-structured populations (or demographic genetics - a topic generally avoided in population genetics texts) and their discussion on the evolution of aging is another unique feature of this book. In the last chapter, molecular evolution, and topics such as segregation distortion, transposable elements, antagonistic and epistatic selection are discussed. These ideas are essential to understand threshold traits and age-related expression of complex diseases. My only wish is that the authors presented their perspective on how molecular variation may be orchestrated through the developmental space generating modular phenotypic diversity, although they state that "a truly predictive theory of development" is still lacking.

In conclusion, the Charlesworths have given us a masterful, well-researched, comprehensive, and insightful treatise of extraordinary breadth and deep scholarship. This book is truly a major achievement not only in evolutionary genetics, but also in biology and medicine, as many of these insights will soon become a part of medical practice and public health. This book is suitable for upper-level undergraduates and graduate students and will serve as a cornucopia of ideas for researchers in all branches of biological sciences. Most importantly, all geneticists and most biologists who wish to understand the evolutionary genetic bases of biological diversity must have a copy on their shelves. The book will serve as a brilliant beacon of light on the subject for decades. 